\title{
Analysis of oxidative stress during the menstrual cycle
}

Umberto Cornelli ${ }^{1}$, Gianni Belcaro ${ }^{2}$, Maria Rosaria Cesarone ${ }^{2}$ and Annarosa Finco ${ }^{3 *}$

\begin{abstract}
Background: Few data concerning the oxidative stress (OS) in plasma during the entire menstrual cycle of eumenorrheic women are available.

Methods: OS was assessed in 20 healthy volunteers during the phase of the menstrual cycle by determining the plasmatic hydroperoxides levels (d-ROMs test). The assessment was performed every three days, starting from the first day (t1) up the end of the menstrual phase (t27). Concomitantly, the estrogen (E2) and progestin (P4) levels were determined at the same time intervals.

Results: From a base value (t1) of $284+/-38.0$ CARR.U., which is essentially within the normal range $(<300$ Carratelli units or CARR.U.), the OS levels progressively increased to $378+/-115$ CARR.U. at t15, and then slightly decreased over the subsequent time but with average values $>300$ CARR.U. Analysis of the E2 levels showed that the maximum OS values were noticed near the estrogen peak, while remaining above the base levels, and then decreased during the progestin phase until returning to normal at the end of the menstrual cycle.
\end{abstract}

Conclusions: It may concludes that the healthy women go into OS for $2 / 3$ of the menstrual cycle.

Keywords: Eumenorrheic, Hydroperoxides, d-ROMs test, Estrogens, Progestagens

\section{Background}

Few data on the variation of oxidative stress across the menstrual cycle in eumenorrheic women have been published $[1,2]$ and no statistically significant differences have been detected on markers such as MDA and linoleic peroxidation derivatives. The markers analyzed were related exclusively to lipids peroxidation, whereas the condition of oxidative stress is affecting many other compounds such as proteins, DNA and sugars. These derivatives taken all together can give a more sensible picture of OS, as it is the case of the hydroperoxides levels in plasma which allow a more complete although nonspecific evaluation of OS [3].

Basing the analysis on the local concentration of GSH (reduced glutathione), GSSG (oxidized glutathione), GSHpx (glutathione peroxidase enzyme) as well as MDA (malonyldialdehyde), Serviddio and colleagues were able to evaluate the change in the oxidative conditions [1] with regard to the menstrual cycle phases. These

\footnotetext{
* Correspondence: finco.annarosa@libero.it

${ }^{3}$ Cor Con. International Srl Res Department, Parma, PR, Italy

Full list of author information is available at the end of the article
}

conditions were then correlated with the estrogen $\left(E_{2}\right)$ and progestin hormone levels (P4), as well as the luteinizing hormone $(\mathrm{LH})$ and follicle-stimulating hormone (FSH).

This data shows that the peak OS phase occurs in the central phases of the cycle (late follicular phase and early luteal phase), or rather at the time of ovular maturation and possible implantation. This phase occurs with: a) an increase in the production of GSHpx; b) a reduction in the GSH; c) an increase in the GSSG; d) a substantial stability of the MDA.

This all seems to indicate that the increase in OS corresponds to a compensation by the antioxidant systems so that the MDA concentration (index of lipid peroxidation) remains constant; in other words, the oxidation/ antioxidant protection system tends to be equilibrated for the entire menstrual cycle. The peak OS will correspond to the $E_{2}$ and LH peaks, while the P4 peak seems to correspond with an OS recovery phase.

The increase in the GSHpx actually corresponds to an increase in the oxidation of GSH; therefore, an increase in the enzyme that "consumes" the GSH to transform
C Biomed Central 
the hydrogen peroxide $\left(\mathrm{H}_{2} \mathrm{O}_{2}\right)$, corresponds to a reduction in the GSH itself, with a resulting increase in the GSSG. This all means OS; it is therefore incorrect to consider GSHpx (any type 1 or 3 ) as an index of antioxidant capacity, since it is caused by an increased need to use GSH to confront the OS.

When the GSHpx system, SOD (superoxide dismutase) and CAT (catalase) in the erythrocytes are simultaneously analyzed over the course of the menstrual cycle [4], the levels of GSHpx increase in the late follicular phase and initial luteal phase with respect to the other phases, while the SOD and CAT levels remain essentially constant with no significant cycle dependent changes. Analysis of the relationships with the levels of $\mathrm{E}_{2}$ indicate that the levels of GSHpx are time related; in extreme synthesis, the increase of one is contemporaneous to the increase of the other. On the contrary, the plasmatic levels of $\mathrm{FSH}, \mathrm{LH}, \mathrm{P} 4$, testosterone and androstenedione do not show any time related relationships with those from the erythrocytic GSHpx and no significant variation in SOD and CAT levels.

From what has been observed, it seems that the isolating action of the $E_{2}$ is not the antioxidant action, but is exactly the opposite [5], which will correspond to the cellular activation needed for the increased energy production from the secretion phase, with a subsequent increase in the production of reactive species such as superoxide $\left(\mathrm{O}_{2}^{*}\right), \mathrm{H}_{2} \mathrm{O}_{2}$ and hydroxyl radicals $\left(\mathrm{OH}^{*}\right)$.

If the ovule is not fertilized, the luteal phase is also characterized by an increase in cellular activity and the proliferation of tissue, which precede the end of the cycle, returning the uterus' mucous membrane to the initial cycle condition with the menstrual flow. Locally, the luteal phase occurs with different activations with respect to the estrogen phase, for example, the increase in mucous' viscosity or in vascular components, and so on. It remains, in any case, similar in terms of oxidation, because it is proliferative phase too, although with different types of final products (mucous, vascular efficiencies). This phase is dominated by the production of $\mathrm{P} 4$, which adds to that of the $E_{2}$, and is therefore the highest combined expression of estrogen-progestin steroids.

The plasmatic conditions of OS have never been continuously examined during all phases of the menstrual cycle; it is therefore unknown if that which occurs locally or at the erythrocyte level is also expressed in a similar way in the plasma.

The purpose of this research is to assess the levels of oxidative stress during the normal menstrual cycle. OS condition has been measured by determining the plasmatic hydroperoxides thought d-ROMs test $[6,7]$.

The most common markers for the evaluation of OS are MDA (TBARS), isoprostanes, carbonylated proteins and oxidized DNA. There is no current consensus about which method is the most useful, reliable and specific for the oxidative insult. The suggestion given by some authors is to use more than one test for a more complete evaluation. The choice of hydroperoxides measurement belongs to the consideration that most of the proteins, DNA and lipids once oxidized are transformed into hydroperoxides (ROOH). These compounds are slowly reactive and can transfer the oxidative stress across the cells [8].

A comparative analysis has been done among the most common markers [3] reaching the conclusion that d-ROMs test seems to be the most reliable.

\section{Methods}

20 eumenorrheic female volunteers were enrolled after a written and informed consent. The study received also an institutional review board approval from the ethics committee of the University of Chieti. They were apparently healthy volunteers, most nulliparous (14 of 20), not following any type of contraceptive therapy. The use of any therapy with dietary supplements was considered grounds for exclusion, as was the excessive consumption of alcohol ( $>3$ alcohol units or AU; considering 1 $\mathrm{AU}=120 \mathrm{~mL}$ of wine or $330 \mathrm{~mL}$ of beer or $40 \mathrm{~mL}$ of spirits), tea ( $>3$ cups/day), coffee ( $>3$ cups/day), chocolate $(>50 \mathrm{~g} /$ day). The exclusion criteria was also extended to women with a history of surgery, with gynecological diseases with recent infections or with restrictive diet.

\section{Analysis of oxidative stress (OS)}

The analysis of the OS condition during the menstrual cycle was assessed on the basis of the Reactive Oxygen Metabolites-derived compounds (d-ROMs) test [4,5] which is used to determine the levels of hydroperoxides in plasma (expression of lipid peroxidation). The analysis was assessed on patients fasting since the evening before the exam.

The blood was taken by pricking the finger and collecting 0.15-0.2 $\mathrm{mL}$ of blood in a heparinized microcuvette. The microcuvette was immediately centrifuged $(6000 \mathrm{~g}$ for $1 \mathrm{~min}$ ) in order to isolate the plasma on which the test is performed, within and no longer than $2 \mathrm{~h}$ from being drawn. The levels of hydroperoxides were quantified in Carratelli Units (CARR.U.; 1 CARR.U. $=0,08 \mathrm{mg}$ $\mathrm{H}_{2} \mathrm{O}_{2} / 100 \mathrm{~mL}$ ).

The normal values are $<300$ CARR.U. The test was applied from the first day from the end of the menstrual flow ( $t_{1}$ or start of the new menstrual cycle) and then every three days (indicated as $t_{1}, t_{3}, t_{n . . .} t_{27}$ ), for the rest of the cycle, up until the time the next menstrual flow completely stopped. The time progression of the OS during the menstrual cycle can be defined in this manner. 


\section{Analysis of the $E_{2}$ and $P 4$ levels}

Only 10 volunteers out of 20 patients accepted to repeat the venous blood sampling and on these patients the protocol also included the evaluation of the $E_{2}$ level at times $t_{6}, t_{9}, t_{12}, t_{15}, t_{18}, t_{21}$, as well as the levels of $\mathrm{P} 4$ at $t_{12}$, $t_{18}, t_{21}, t_{24}$, in order to determine the correlation of OS with the secretion and luteal phases of the menstrual cycle.

The levels of $E_{2}$ and P4 were determined using commercially available kits (Estradiol: Catalog No KE2D1; Progesterone: TKPG1; Inter Medico Markham, OntarioCanada). The detection limits for these tests were $5 \mathrm{pg} /$ $\mathrm{mL}$ for $\mathrm{E}_{2}$ and $0.1 \mathrm{ng} / \mathrm{mL}$ for $\mathrm{P} 4$, with variation coefficients of $13.1 \%$ for $E_{2}$ and $6.5 \%$ for P4. The blood for these hormones ( 2 aliquots of $5 \mathrm{ml}$ each) was taken from the arm vein in heparinized test tubes. All blood withdrawals were done in the morning between 8:00 and 9:30 a.m. with analytical determination within that same day. The volunteers were told to avoid eating large amounts of food the night before the laboratory tests.

\section{Statistical analysis}

From previous experience on d-ROMs test [5] for $\alpha=0.05$ and $1-\beta=0.9$ a sample of 12 cases is sufficient to discriminate groups with a power $>0.9$. The average values and dispersion parameters $(\mathrm{SD})$ were calculated on all data. The comparison between the base values and those at the various observation times were done using Randomized Block Design Anova, the multiple comparisons were calculated using "Dunnett's Two-Sided Multiple Comparison Test vs $t_{1}$ for $d-R O M s$ test, $v s t_{6}$ for $E 2$ and vs $t_{12}$ for P4. The relationships between the d-ROMs test and $E_{2}$ or P4 levels based on the parametric correlation test.

\section{Results}

Despite the limited number of patients $(n=20)$, all volunteers finished the study. The general characteristics of the volunteers are reported in Table 1 . The OS increase (see Table 2) begins on average between $t_{6}$ and $t_{24}$, and the hydroperoxide levels return to the base values from the start of the new cycle at $t_{27}$.

The concomitance of the maximum increase of hydroperoxides corresponds to a delay from three to six days with respect to the estrogen peak. The rising of the $\mathrm{P} 4$

\section{Table 1 General characteristics of the volunteers} (Mean \pm SD)

\begin{tabular}{lc}
\hline & Mean +/- SD \\
\hline Patients & 20 \\
Age $(\mathrm{yrs})$ & $30.8+/-5.66$ \\
Weight $(\mathrm{Kg})$ & $63.2+/-6.66$ \\
Height $(\mathrm{m})$ & $1.67+/-0.07$ \\
BMl $\left(\mathrm{Kg} / \mathrm{m}^{2}\right)$ & $22.6+/-1.58$ \\
\hline
\end{tabular}

levels instead seems to cause a slight reducing effect on the OS when at its hematic peak $\left(t_{21}\right)$ but not before.

As can be seen from the average values, the increase in the CARR.U. with respect to those from the start of the cycle reach the condition defined as OS $(>300$ CARR.U.) from $t_{12}$ to $t_{24}$.

With regard to that noted in the literature [9], the $E_{2}$ levels reach a peak between $t_{9}$ and $t_{12}$ while the P4 levels significantly increase between $t_{18}$ and $t_{21}$. Our evaluations extended to $t_{24}$, which is the moment when the inflection of the P4 levels was observed.

No correlations between the d-ROMs test and $E_{2}$ levels were observed; this seems to indicate that the increase in systemic oxidation is not determined by just $E_{2}$, but also by other factors that could not be identified at our experimental conditions (hypothetically referring to levels of LH, FSH, relaxin, etc.).

\section{Discussion}

Browne et al. [2] end up with the conclusion that no significant variation of the OS markers can be shown during the menstrual cycle. However the data were limited to 9 cases with a large variation of BMI (ranging between 20.9 and 34.7) and also the inter-individual coefficient of variation of the OS markers were very high (from 30 to 56\%). This means that the power of the analysis was very limited. In previous trials the coefficient of variation of d-ROMs with almost a similar sampling (12 cases) was $<10 \%$, as a consequence we may suppose that the hydroperoxides measure can be more reliable and early in detecting the condition of OS.

From that observed, a woman is in an OS condition for a good portion of the menstrual cycle, starting near her estrogen peak and hydroperoxides tend to decrease slightly when progestin hormone increases, which however does not allow normal levels to be reached. This fact puts all of the findings made up to now into question in terms of OS in pre-menopausal women.

Therefore, the OS indexes for fertile women should be reviewed in relation to the menstrual period.

If OS relates to a particular moment of the menstrual cycle, its correlations with the various pathologies should be corrected in relation to this "oxidative cyclicity". Obviously, that seen for lipid type hydroperoxides may not apply to the other OS markers, such as isoprostanes or DNA oxidation products (8-OHdG for example) and proteins (carbonylated proteins); nevertheless, at least in part, some of these derivatives also have a hydroperoxide component.

All the hypotheses for the reason for this cyclic algorithm are to be investigated. We know that both the estrogen and the progestin phases have an activation phase; the proliferation phase involves an increase in the production and consumption of energy, which continues 
Table 2 d-ROMs test, $E_{2}, P 4$ values at various times in healthy volunteers (average \pm SD)

\begin{tabular}{|c|c|c|c|c|c|c|c|c|c|c|}
\hline & $\mathrm{t} 1$ & t3 & t6 & t9 & $\mathrm{t} 12$ & t15 & $\mathrm{t} 18$ & t21 & $\mathrm{t} 24$ & t27 \\
\hline \multirow[t]{3}{*}{ d-ROMs CARR.U. } & 284 & 273 & 284 & $299^{a}$ & $336^{a}$ & $378^{a}$ & $326^{a}$ & $315^{a}$ & $323^{a}$ & 276 \\
\hline & $+/-$ & $+/-$ & $+/-$ & $+/-$ & $+/-$ & $+/-$ & $+/-$ & $+/-$ & $+/-$ & $+/-$ \\
\hline & 38.0 & 47.0 & 34.6 & 31.3 & 66.8 & 115.6 & 66.4 & 56.3 & 51.1 & 44.8 \\
\hline \multirow[t]{3}{*}{$E_{2} \mathrm{pg} / \mathrm{mL}$} & & & 99 & $222^{b}$ & $195^{\mathrm{b}}$ & $134^{\mathrm{b}}$ & $157^{\mathrm{b}}$ & 126 & & \\
\hline & & & $+/-$ & $+/-$ & $+/-$ & $+/-$ & $+/-$ & $+/-$ & & \\
\hline & & & 33.7 & 34.2 & 39.9 & 36.2 & 36.9 & 38.4 & & \\
\hline \multirow[t]{3}{*}{ P4 ng/mL } & & & & & 1.7 & & $6.3^{c}$ & $13.0^{c}$ & $5.9^{c}$ & \\
\hline & & & & & $+/-$ & & $+/-$ & $+/-$ & $+/-$ & \\
\hline & & & & & 0.54 & & 3.0 & 6.2 & 3.8 & \\
\hline
\end{tabular}

${ }^{a}$ Dunnett's Two-Sided Multiple Comparison Test, $\mathrm{p}<0.05 \mathrm{t}_{\mathrm{n}} \mathrm{Vs}_{\mathrm{s}}$

${ }^{b}$ Dunnett's Two-Sided Multiple Comparison Test, $p<0.05 t_{n} V_{s} t_{6}$.

'Dunnett's Two-Sided Multiple Comparison Test, $\mathrm{p}<0.05 \mathrm{t}_{\mathrm{n}} \mathrm{Vs}_{12}$.

into the rather luteal phase of vascular development (vascular space) with a substantial production of nitric oxide $\left(\mathrm{NO}^{*}\right)$.

Nitric oxide, besides having a radical nature, is an important mediator for vasodilatation, the relaxation of the myometrium, anti-aggregation action and angiogenic stimulation which occurs by means of VEGF (vascular endothelial growth factor); this in turn stimulates $\mathrm{NO}$ synthase (NOS) [10], indicating that the $\mathrm{NO}^{*}$ is trying to maintain its level with the self-induction of NOS.

This synthesis, even though it occurs in all menstrual phases as eNOS (endothelial) and iNOS (induced), increases slightly with progestin stimulus [11]. This entire system must be counterbalanced in a very precise manner.

Locally, there is also an increase in the SOD that coincides with this increase in the synthesis of NO'. SOD, carrying out the dismutation of $\mathrm{O}_{2}^{*}$ (superoxide), to form $\mathrm{H}_{2} \mathrm{O}_{2}+\mathrm{O}_{2}$, inhibits the $\mathrm{NO}^{-}+\mathrm{O}_{2}^{*}$ reaction that tends to produce the $\mathrm{ONOO}^{-}$ion (peroxynitrite) which, besides having a strong oxidizing strength, removes $\mathrm{NO}^{\circ}$ and limits its availability. Nevertheless, the high level of available $\mathrm{NO}^{\circ}$ can however react with $\mathrm{O}_{2}^{*}$, since the reaction between the two is by far the fastest in the body $(1 \times$ $\left.10^{-9} \mathrm{sec}\right)$ and can generate OS. It is therefore not surprising that the vectorial result of the antioxidant and oxidant production, action of the progestin phase stabilizes on an OS scale. That which occurred in terms of d-ROMs values was seen in all cases analyzed, therefore the data indicating that OS conditions are present for approximately $2 / 3$ of the menstrual cycle phases is considered "solid".

The connection between OS and the $E_{2}$ and P4 levels is also confirmed by the previous observations, since we know that the levels of hydroperoxide in women taking birth control pills increase significantly $[12,13]$. This fact must clearly be confirmed through specific studies, but tends to discredit the hypothesis that exogenous estrogens have an antioxidant action [14,15].
The opposite instead seems to be true. Hypothetically speaking, the action of the estrogens may be similar to that which occurs during exertion [16]. In this condition, the LDLs are oxidized but are also more easily up-taken by the blood receptors, or kept under control and partially "diminished" by the muscular antioxidant systems. Same has been described by other authors [17] that suggest estradiol promote the oxidation of LDL and promotes their clearance from the liver. If however their quantity increases, even at the rate of an inefficient antioxidant system, they are subject to vascular uptake (sub-endothelial) triggering the atherosclerotic inflammatory process. The sub-endothelial will in fact be subject to the action of myeloperoxidase (MPO), which are oxidant-producing enzymes.

This justifies the reduction of the cholesterol and the LDL in premenopausal women, which should not be interpreted as greater efficiency of the LDL blood receptors, but as an increased uptake of LDL thanks to their partially oxidized condition (keep in mind that there is a minimum repairable oxidation limit above which triggers the macrophage reaction).

This oxidative balance, already modified under physiological conditions of normal estrogen/progestin secretion (thus during a normal menstrual phase) can lead to an excess of oxidant production when birth control (BC) therapy is followed. This phenomenon is understandable, since any oral administration of estrogen/progestin generates a hematic peak in both, which is not on a physiological scale and therefore the OS can become permanent.

It belongs to previous observations that anti-proteases in general are more sensible than proteases to oxidation, due to presence of an higher amount of methionine in anti-proteases [18]. Since some proteases such as thrombin are pro-coagulant whereas anti-proteases are anticoagulant (serpins), the tendency of an increase of OS will be a pro-thrombotic status. 
A protection mechanism from these thrombotic phenomena, besides the maintaining of the vascular patency of the endometrium and its regular distribution of blood, could originate from the presence of a special peptide hormone in women, relaxin (RLX), which could act as a powerful stimulant for the antioxidant systems [19].

Relaxin is a polypeptide with a MW of $6 \mathrm{KDa}$ and is composed of two peptide chains stabilized both internally and between each other by S-S bridges [19]. It is mainly secreted by the corpus luteum and strategically inhibits alterations in the endometrial flow, to prevent ischemia and reperfusion phenomena, both during the cycle's proliferation phase and then during the eventual pregnancy. The ischemia and reperfusion phenomenon causes OS, both by the production of ROS and by chemotactic stimulation.

The action of the RLX works by over-regulating the iNOS and eNOS, besides the angiogenesis in particular but not exclusively - in the endometrium; this stimulus occurs in both arterial and venous vessels (thus also in vessels without smooth muscle). RLX is active at nanomolecular concentrations, which therefore can be obtained during the endometrial phases by the ovular implantation and by pregnancy. The angiogenic action is typical of RLX and occurs through the release of VEGF and also bFGF (basic fibroblast growth factor).

An important aspect of the action of RLX is the inhibition of the endothelial adhesiveness of the inflammatory cells (lymphocytes, macrophages) as well as platelet aggregation.

Overall, RLX substantially influences the perfusion and hematic distribution in the endometrium, giving it the ideal flow conditions and performing an antiinflammatory and anti-thrombotic action and opposes the effects of OS; it could therefore perform a protective action against $\mathrm{OS}$.

\section{Conclusions}

Healthy eumenorrheic women go into OS for $2 / 3$ of the menstrual cycle. Oxidative stress is however a physiological condition for women of child-bearing age. This implies that an over-regulation of the OS defense mechanisms is also present. Therefore, all the OS markers should be studied during the various phases of the cycle and parameterized to this phenomenon.

\section{Competing interests}

The authors declare that they have no competing interests.

\section{Authors' contributions}

Research was designed by CU; BG and MRC were responsible for the volunteers selection and for the $E_{2}$ and $P 4$ determination. The $d-R O M s$ on plasma samples was carried out by FA at the Cor. Con. International Srl Research Labs, that provided all the analytical reagents also. All authors read and approved the final manuscript.

\section{Acknowledgments}

We are profoundly grateful to Professor Martino Recchia for the statistical analysis of the data. No financial or contractual agreement that may cause conflict of interest or be perceived as causing are in place. No works are being concurrently published or reviewed that are relevant to the manuscript. The corresponding author and all of the authors have read and approved the final submitted manuscript. No portion of this work has been or is currently under consideration for publication elsewhere. No portion of this manuscript, other than the abstract, has been published or posted on the internet.

\section{Author details}

'Loyola University School of Medicine-Chicago, Chicago, USA. ${ }^{2}$ University of Chieti, Chieti, Italy. ${ }^{3}$ Cor Con. International Srl Res Department, Parma, PR, Italy.

Received: 19 April 2013 Accepted: 29 July 2013

Published: 2 August 2013

\section{References}

1. Serviddio G, Loverro G, Vicino M, Prigigallo F, Grattagliano I, Altomare E, Vendemiale G: Modulation of endometrial balance during the menstrual cycle: relation with sex hormones. J Clin Endocrinol Metab 2002, 87:2843-2848.

2. Browne RW, Bloom MS, Schisterman EF, Hovey K, Trevisan M, Wu C, Liu A, Wactawski-Wende J: Analytical and biological variation of biomarkers of oxidative stress during the menstrual cycle. Biomarkers 2008, 13:160-183.

3. Cornelli U, Belcaro G, Finco A: The oxidative stress balance measured in humans with different markers, following a single oral antioxidants supplementation or a diet poor of antioxidants. J Chem Dermatol Sci Appl 2011, 1:64-70

4. Massafra C, Gioia D, De Felice C, Picciolini E, De Leo V, Bonifazi M, Bernabei A: Effects of estrogens and androgens on erythrocyte antioxidant superoxide dismutase, catalase and glutathione peroxidase activities during the menstrual cycle. J Endocrinol 2000, 167:447-452.

5. Santanam N, Shern-Brewer R, McClatchey R, Castellano PZ, Murphy AA, Voelkel S, Parthasarathy S: Estradiol as an antioxidant: incompatible with its physiological concentrations and function. J Lipid Res 1998, 39:2111-2118.

6. Cesarone MR, Belcaro G, Carratelli M, Cornelli U, De Sanctis MT, Incandela L, Barsotti A, Terranova R, Nicolaides A: A simple test to monitor oxidative stress. Int Angiol 1999, 18:127-130.

7. Cornelli U, Terranova R, Luca S, Cornelli M, Alberti A: Bioavailability and antioxidant activity of some food supplements in men and women using the d-ROMs test as a marker of oxidative stress. J Nutr 2001, 131:3208-3211.

8. Brigelius-Flohè R, Flohé L: Basic principles and emerging concepts in the redox control of transcription factors. Antioxid Redox Signal 2011, 15:2335-2381.

9. Loose DS, Stancel GM: Estrogens and Progestins. In Goodman \& Gilman's the pharmacological basis of therapeutics. 11th edition. Edited by Brunton LL, Lazo JS, Parker KL. New York: McGraw-Hill; 2006:1541-1571.

10. Fukumura D, Gohongi T, Kadambi A, Izumi Y, Ang J, Yun CO, Buerk DG, Huang PL, Jain RK: Predominant role of endothelial nitric oxide synthase in vascular endothelial growth factor-induced angiogenesis and vascular permeability. Proc Soc Acad Sci USA 2001, 98:2604-2609.

11. Cameron IT, Campbell S: Nitic oxide in the endometrium. Human Repr Update 1998, 4:565-589.

12. Foidart JM, De Groote D, Claesen J, Gerday C, Balteau B, Pintiaux A, Perrier d'Hauterive S: Effect of oral contraception with ethiylestradiol and drospirenone on oxidative stress in women 18-35 years old. Contraception 2009, 80:187-193.

13. Pincemail J, Vanbelle S, Gaspard U, Collette G, Haleng J, Cheramy-Bien JP, Charlier C, Chapelle JP, Giet D, Albert A, et al: Effect of different contraceptive methods on the oxidative status in women aged 40-48 years from ELAN study in the province of Liège- Belgium. Human Reprod 2007, 22:2335-2343.

14. Sack MN, Rader DJ, O Cannon R: Oestrogens and inhibition of oxidation of low-density lipoproteins in postmenopausal women. Lancet 1994, 343:269-270.

15. Sugioka K, Shimosegawa Y, Nakano M: Estrogerns as natural antioxidants of membrane phospholipid peroxidation. FEBS 1987, 210:37-39.

16. Shern-Brewer R, Santanam N, Wetzstein C, White-Welkley J, Parthasarathy S: Exercise and cardiovascular disease a new perspective. Arteriosc Thromb Vasc Biol 1998, 18:1181-1187. 
17. Chiang K, Parthasarathy S, Santanan N: Estrogen, neutrophils oxidation. Life Sci 2004, 75:2425-2438.

18. Swaim MW, Pizzo SV: Methionine sulfoxide and the oxidative regulation of plasma proteinase inhibitors. J Leuk Biol 1988, 43:365-379.

19. Bani D: Relaxin as a natural agent for vascular health. Vasc Health Risk Manag 2008, 4:515-524.

doi:10.1186/1477-7827-11-74

Cite this article as: Cornelli et al: Analysis of oxidative stress during the menstrual cycle. Reproductive Biology and Endocrinology 2013 11:74.

\section{Submit your next manuscript to BioMed Central} and take full advantage of:

- Convenient online submission

- Thorough peer review

- No space constraints or color figure charges

- Immediate publication on acceptance

- Inclusion in PubMed, CAS, Scopus and Google Scholar

- Research which is freely available for redistribution 